\author{
Kouame $\mathrm{N}^{1 \star}$, Bakary $\mathrm{YN}^{2}$, Manewa \\ FS $^{1}$, Gaimou BP ${ }^{1}$, Agoda $\mathrm{AK}^{2}$, Ngoan \\ Domoua $\mathrm{AM}^{1}$ and $\mathrm{N}^{\prime}$ gbesso $\mathrm{RD}^{1}$ \\ ${ }^{1}$ Department of Radiology, University Hospital of \\ Yopougon, Abidjan 21 BP 632 21, Abidjan, Ivory \\ Coast \\ ${ }^{2}$ Department of Radiology, Sylvanus Olympio. BP 57 \\ Lome, Togo \\ Dates: Received: 21 December, 2015; Accepted: \\ 08 January, 2016; Published: 09 January, 2016 \\ *Corresponding author: Dr Kouame N, Senior \\ lecturer in diagnostic radiology and medical imaging, \\ University Felix Houphouet Boigny, Abidjan, Ivory \\ Coast. 21 BP 2716 Abidjan 21, Tel: 22505190002 ; \\ E-mail: kngoran@yahoo.fr \\ www.peertechz.com
}

Keywords: Abdominal pelvic mass; Abdominal pelvic CT; Giant renal cyst; Bladder distention; Geriatrics

\author{
Case Report
}

\section{Abdominal-Pelvic Masses in Geriatrics: A Report about 02 Cases Explored at the Computed Tomography (CT)}

\section{Introduction}

Abdominal-pelvic masses are often described in children [1,2]. In adults or in the elderly, these masses are less bulky and are rarely documented and therefore they are rarely the cause of abdominal pelvic distension. Regardless of the age of discovery, the abdominalpelvic masses make fear malignancy. They thus require the initiation of a very important diagnostic arsenal, sometimes abusive and costly condemning the less affluent patients to opt for traditional medicine which is unlikely even suicidal. Yet CT alone can be sufficient in our view to make the diagnosis and should be of easy indication in the elderly. We report 02 cases of abdominal pelvic masses of benign and/or mundane causes encountered in subjects aged 76 and 91 years explored on CT at the University Hospital of Yopougon (Ivory Coast) and at the University Hospital Sylvanius Olympio in Lomé ( Togo). Our objective was to insist on the contribution of the scanner in the diagnosis of large abdominal-pelvic masses in the elderly and to describe two causes of abdominal-pelvic mass in the elderly in subSaharan Africa.

\section{Observation no 1}

$\mathrm{K}$ is a76 year-old male patient. He was referred to the radiology department of the University Hospital Sylvanus Olympio to explore a large abdominal pelvic mass accompanied by left abdominal and lumbar pain in a context of anemia and high blood pressure. The CT scan was performed using a multi-cut unit of General Electrics. An abdominal pelvic acquisition in spontaneous contrast and another after iodinated contrast medium injection were performed. A large fluid mass (Figure 1) of $237 \mathrm{~mm}$ in diameter with a thin wall was highlighted in the left kidney. It presented a thin full partition. There was no contrast enhancement in the mass, wall or internal vegetation. The left kidney was rolled without dilatation of its excretory cavities and surrounding organ and vascular structures were repressed. The

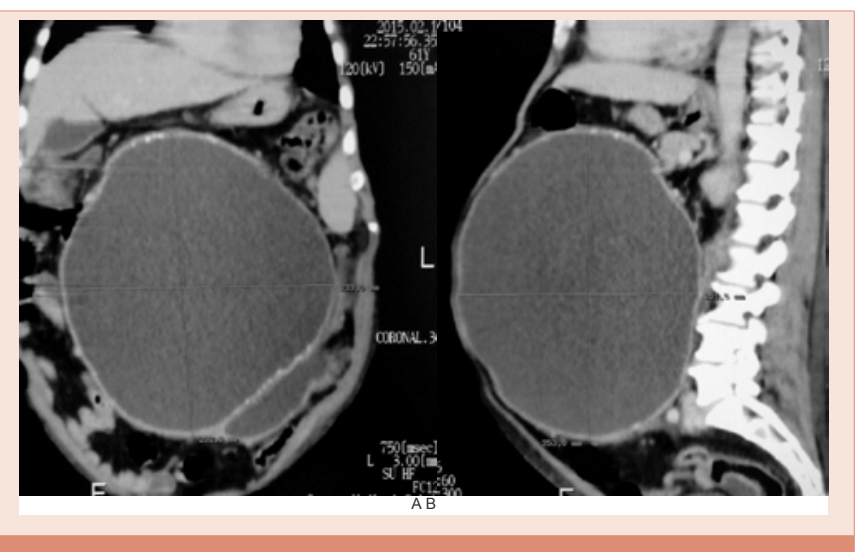

Figure 1: Abdominal-pelvic CT, coronal (A) and sagittal $(B)$ sections, use iodinated contrast-enhanced, showing a large fluid mass (Figure 1) of 237 $\mathrm{mm}$ in diameter with a thin wall was highlighted in the left kidney. It presented a thin full partition. There was no contrast enhancement in the mass, wall or internal vegetation: giant left renal cyst. 
contralateral kidney was normal. The examination concluded that it was a giant renal cyst type 2 of Bosniak (Table 1). The patient underwent a cystectomy and a left nephrectomy. The pathological examination of the surgical specimen was in favor of benignancy.

\section{Observation no 2}

YKJ is a 91 year-old male patient with no history of known prostate adenoma. He was taken from his village to the emergency unit of the University Hospital of Yopougon in a context of abdominal distension and abdominal-pelvic pain associated with profuse sweating and hypertension. The clinical examination revealed a median large abdominal pelvic mass of elastic consistency. A CT scan is required by the emergency physician. It was performed using a Toshiba device with 64 strips with and without iodinated contrast injection. Computed tomography has objectified a large distended bladder (267 $\mathrm{mm}$ in diameter) on heterogeneous prostatic hypertrophy (Figure 2). The patient has simply benefited from the setting of a bladder catheter that solved his problem. He was then transferred to the urology department for treatment of his prostatic hypertrophy.

\section{Comments}

Abdominal-pelvic masses are more common in children [1] Their causes are dominated by malignant tumors particularly nephroblastoma and neuroblastoma [3]. In the adult female, it is routinely met with voluminous abdominal-pelvic masses due to uterine fibroadenomas (the black race is an important risk factor) and large ovarian cysts [4-6]. In the adult male even in both sexes, apart from the voluminous splenomegalies of type IV, the masses that are large enough to be both abdominal and pelvic are rare. It is the same for the elderly. Our 02 patients were males. They are 76 and 91 years old and are therefore the elderly or geriatricians, given that the subject who is called geriatrician is the subject whose age exceeds 65 years, according to $\mathrm{WHO}$, and 75 years according to some authors $[7,8]$. It is an age group marked by behavioral problems and slower ideation usually caused by brain deficiencies caused more by episodes of hypertension. Our 02 patients were known hypertensive persons. The CT indications were exploration of abdominal-pelvic masses. This is about fluid masses for which ultrasound diagnosis would have been easy had it not been their large size 237 and $267 \mathrm{~mm}$ in diameter.
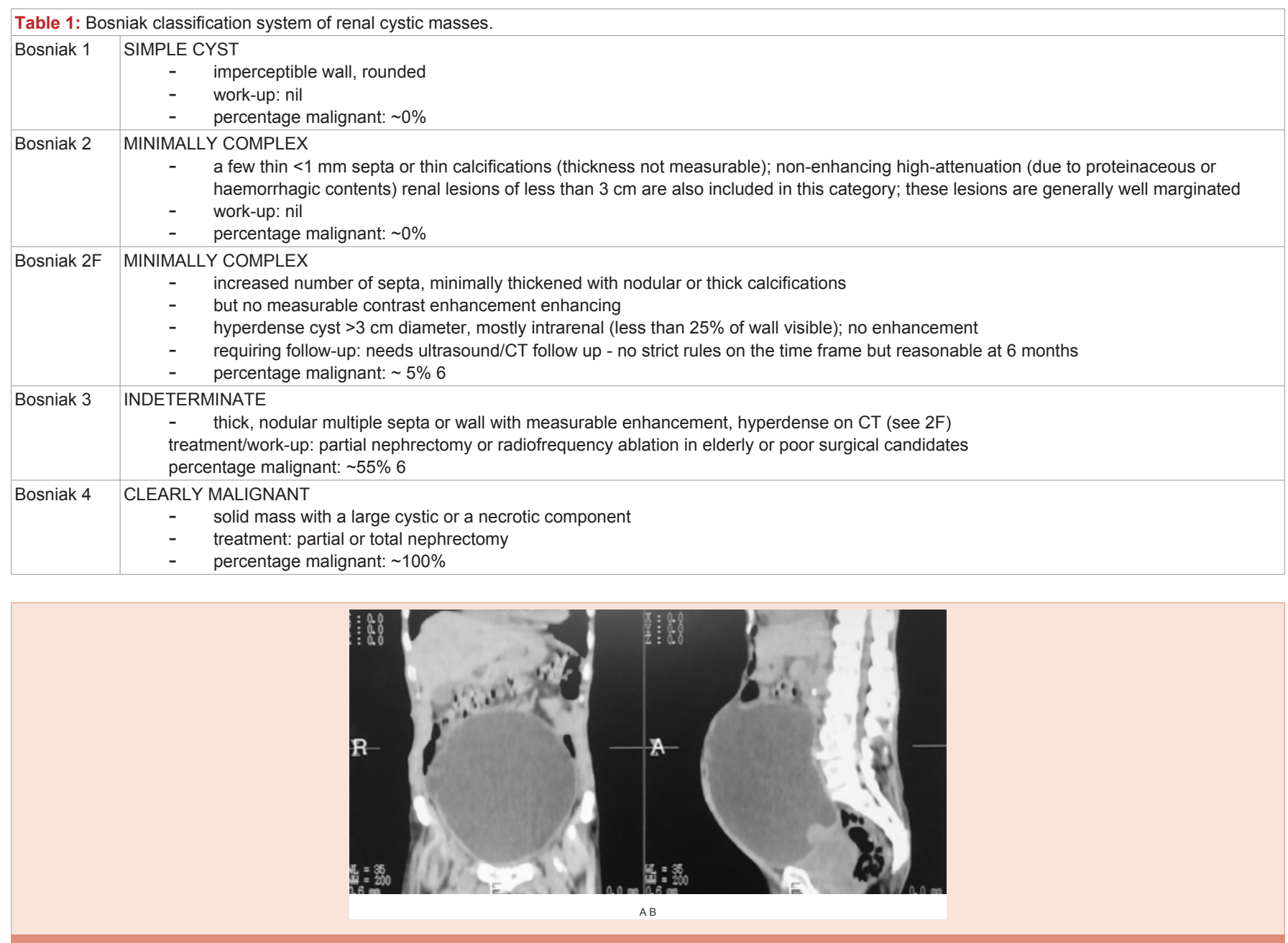

Figure 2: Abdominal-pelvic CT, coronal (A) and sagittal (B) sections, use iodinated contrast-enhanced objectifiying a large distended bladder (267 mm in diameter) on heterogeneous prostatic hypertrophy. 
It is well known that ultrasound is not contributory when the mass is very large. The multislice scanner though very radiant appears as the best imaging method [9]. It allows to explore a large volume and do multiplanar reconstructions thus facilitating the diagnosis of large masses in the absence of MRI. With its spatial resolution superior to MRI [10] and the possibility to measure the densities and to study the vascular kinetics of masses, computed tomography is an excellent imaging examination for explorations in geriatrics. It helped to make organ diagnosis in both cases. In the first case, thanks to Bosniak classification [11], the giant renal cyst was suggested Benign and confirmed at pathology. In the second case, it was a distended bladder with a banal (catheterization) and immediate management. Both etiologies we report have in common the fact that they are of urological origin. Our observations are corroborated by the literature that describes the predominance of urological etiologies during the exploration of abdominal-pelvic masses $[9,12]$, in the male subject.

\section{Conclusion}

Large abdominal-pelvic masses in geriatrics are usually benign and of urologic origin. The scanner diagnostic support and allows the initiation of a treatment sometimes banal as the setting of a urinary catheter. Indications of CT in geriatrics must be as wide as possible even in the absence of the possibility of iodinated contrast medium injection.

\section{References}

1. Sarnacki S, Brisse H, Schleiermacher G, Doz F (2009) Conduite à tenir devant la découverte d'une tumeur abdominale de l'enfant. mt pédiatrie 12 29-38.
2. Holbrook J, Schneller C, Lapping-Carr G, Hageman JR, Lo A (2015) A 3 -year-old female with abdominal pain, vomiting and an abdominal mass. pediatr Ann 44: e6-9.

3. Plantaz D, Pasquier D, Dyon JF, Favrot M, Bost M (2001) Neuroblastomes: aspects cliniques, biologiques et thérapeutiques actuels. Médecine Nucléaire - Imagerie fonctionnelle et métabolique 25: 207-212.

4. Funaki K, Fukunishi H, Tsuji Y, Maeda T, Takahashi T (2013) Giant cystic leiomyoma of the uterus occupying the retroperitoneal space. J Radiol Case Rep 7: 35-40.

5. Dubuisson J, FehlmannA (2015) Comment je fais... le traitement d'un kyste ovarien géant présumé bénin par cœlioscopie. Gynécologie obstétrique \& fertilité 43: 81-83.

6. Casanelli JM, Keita M, N'Dri, Aboua G, Moussa B, et al. (2010) kyste géant de l'ovaire en Côte d'ivoire. Médecine Tropicale 70:122.

7. Blickle JF, Attali JR, Barrou Z, Brocker P, DeRekeneire N, et al. (1999) Le diabète du sujet âgé. Diabetes \& Metabolism 25: 84-93.

8. Chabot F (2007) Insuffisance respiratoire aiguë du sujet âgé: commentaires. Revue des Maladies Respiratoires 24: 41-48.

9. La Fianza A, Campani R, Dore R (1994) Computerized tomography in the differential diagnosis of non-gynecologic abdomino-pelvic masses. Radiol Med 88: 472-477.

10. Zins M, Fontanelle L, Lenoir S, Bouza N, Strauss C, et al. (2003) Scanner multidétecteur face à l'IRM dans la pathologie du pancréas. Journal de radiologie 84: 484-496

11. Thomas F Whelan (2010) Guidelines on the management of renal cyst disease. Can Urol Assoc J 4: 98-99.

12. Kumar S, Jayant K, Barapatra Y, Rani J, Agrawal S (2014) Giant Urinary Bladder Diverticula presenting as Epigastric Mass and Dyspepsia. Nephrourol Mon 6: e18918. 